\section{Herausfordernd und lohnend}

\author{
Unternehmen und Gesellschaft sind derzeit noch nicht nachhaltig. Dennoch ist \\ dieses Leitbild für die Deutsche Telekom eine lohnende Zukunftsalternative. Der \\ Markt erlaubt zwar keine Alleingänge, aber die pro-aktive gleichberechtigte $\mathrm{Be}$ - \\ rücksichtigung der ökologischen und sozialen Dimension zahlt sich für das Unter- \\ nehmen auch wirtschaftlich langfristig aus.
}

$\mathrm{N}$

Von Ignacio Campino

derner Unternehmen geworden. Sie lässt sich definieren als eine Wirtschaftsform, die ökonomische, soziale und ökologische Belange gleichberechtigt berücksichtigt und darüber hinaus die Entwicklungsmöglichkeiten der ärmeren Länder und der zukünftigen Generationen nicht einschränkt. Jedoch sieht die Situation im Alltag - sei es in den Unternehmen oder in der Gesellschaft insgesamt - völlig anders aus. Ökonomische Aspekte dominieren, auch wenn in Deutschland eine starke soziale Komponente unsere Handlungen beeinflusst. Der vom ehemaligen Bundeskanzler Ludwig Erhard geprägte Begriff der Sozialen Marktwirtschaft ist immer noch gïltig. Darüber hinaus ist auch das Umweltrecht hier im Lande stark entwickelt. Aber dennoch befinden wir uns heute noch weit entfernt von einer nachhaltigen Gesellschaft. Der Hauptgrund liegt vor allem im Konsumverhalten der Industrienationen mit dem daraus folgenden hohen Ressourcenverbrauch.

Auch die Deutsche Telekom erkennt im Leitbild der Nachhaltigkeit eine Zukunftsalternative, für die es lohnt, sich einzusetzen. Allerdings ist für ein Unternehmen der Alleingang in Richtung Nachhaltigkeit nicht möglich, will es sich nicht in kurzer Zeit aus dem Markt katapultieren. Die Strategie muss also sein, sich im Rahmen des Machbaren der Nachhaltigkeit anzunähern und die Grenze allmählich - gemeinsam mit anderen Unternehmen und mit weiteren wichtigen Akteuren in der Gesellschaft - auszuweiten. Wir müssen heute akzeptieren: Der Weg ist das Ziel.

Der gewaltige Umwandlungsprozess der Deutschen Telekom von der alten Behördenstruktur zu einem internationalen High-tech-Unternehmen der Telekommunikation bietet besondere Chancen, der Nachhaltigkeit näher zu kommen. Sie liegen unter anderem in der Tradition der alten Deutschen Bundespost bezüglich der hervorra- genden Aus- und Fortbildungsprogramme und des sehr flexiblen Einsatzes des Personals über verschiedene Fachgebiete und Organisationseinheiten hinweg, sowie auch im hohen Umweltbewusstsein der Beschäftigten.

Im Folgenden wird vor allem auf die sozialen und ökologischen Faktoren eingegangen.

\section{- Soziale Verantwortung}

Das größte Potenzial der Deutschen Telekom zum erfolgreichen Agieren im internationalen Markt sind ihre Mitarbeiter. Auch wenn aus Wettbewerbsgründen in wichtigen Bereichen des Unternehmens eine Personalreduktion erfolgen musste, hat sich das Unternehmen verpflichtet, bis zum Jahre 2004 keine betriebsbedingten Kündigungen auszusprechen. Die erforderliche Personalreduktion erfolgt sozialverträglich. Aufgrund technischer Innovationen und der konsequenten Marktausrichtung des Unternehmens ist eine Verschiebung des Personalbedarfes im Unternehmen aufgetreten. Während der Personalbedarf für den Betrieb des Netzes gesunken ist, ist ein Mehrbedarf im Vertrieb und Kundenservice sowie auch bei den sogenannten neuen Diensten, wie Internet oder hochwertige Call-Center zur Kundenbetreuung, entstanden.

Zur Befriedigung des Bedarfs des internen Arbeitsmarktes wurde ein großes Projekt zur Personalumsetzung innerhalb des Unternehmens gestartet. Damit erfüllt es seine Selbstverpflichtung zur sozialen Verantwortung und verhindert gleichzeitig

\begin{tabular}{|c|}
\hline Einen Überblick über alle bisher \\
erschienen Hefte des Informationsdienstes \\
"Ökologisches Wirtschaften" \\
finden Sie auf unseren \\
Internetseiten unter der Adresse: \\
www.oekom.de \\
Wir freuen uns auf Ihren Besuch! \\
\hline
\end{tabular}

einen Aderlass an Know-how und wertvollen Erfahrungen. Kern des Programms ist eine adäquate Umschulung der Mitarbeiter in Vorbereitung auf die neuen Aufgaben. Das Qualifizierungsprogramm beschränkt sich nicht nur auf das umzusetzende Personal, sondern berücksichtigt alle Mitarbeiter, einschließlich der Führungskräfte. Die neuen Anforderungen des Marktes müssen von allen Beschäftigten erfïllt werden.

\section{- Umweltleistung verbessern}

Die Deutsche Telekom ist eines der jüngsten Großunternehmen Deutschlands. Nichtsdestotrotz hat sie in kurzer Zeit beispielhafte Umweltleistungen auf Weltniveau erbracht. Diese wurden in verschiedenen externen öko-Ratings bestätigt.

Der Vorstand hat gerade das Umweltprogramm für den Zeitraum 2001 bis 2004 beschlossen. Darin ist ein wichtiger Satz enthalten: ,Wir wollen ein nachhaltiges Unternehmen in einer nachhaltigen Gesellschaft werden." Unser Anspruch ist, alle Prozesse im Unternehmen aus der Sicht des Umweltschutzes zu beleuchten, mit dem Ziel einer kontinuierlichen Verbesserung der Umweltleistungen, um uns der Nachhaltigkeit schrittweise anzunähern.

Unsere Strategie aus der Sicht des Schutzes der natürlichen Ressourcen berücksichtigt vier Aspekte:

1. Einhaltung der gültigen Gesetzgebung

Das gesetzliche Regelwerk entwickelt sich weiter und die Aktivitäten im Unternehmen müssen ständig angepasst werden. Damit wird sichergestellt, dass in allen Aktivitäten Rechtssicherheit sichergestellt ist.

2. Aufbau, Pflege und weitere Entwicklung des prozessorientierten Managementsystems mit dem Ziel, Einspar- und Rationalisierungspotenziale zu erkennen und zu nutzen

Nachdem in allen Organisationseinheiten des Unternehmens das Qualitätsmanagementsystem nach ISO 9001 implementiert wurde, wurde in allen Einheiten mit einer größeren Umweltrelevanz - hierzu zählen vor allem die Zentrale des Unternehmensbereiches Netze, die Technikniederlassungen und die Logistikzentren - Umweltmanagementsysteme nach ISO 14.001 eingeführt und mit dem bereits vorhandenen Qualitätsmanagementsystem integriert. Bei den Einheiten mit einer geringeren Umweltrelevanz - hierzu zählen vor allem die Kundenniederlassungen - wurde auf die Implementierung dieser Norm auf Grund des ungünstigen KostenNutzen-Verhältnisses verzichtet. 


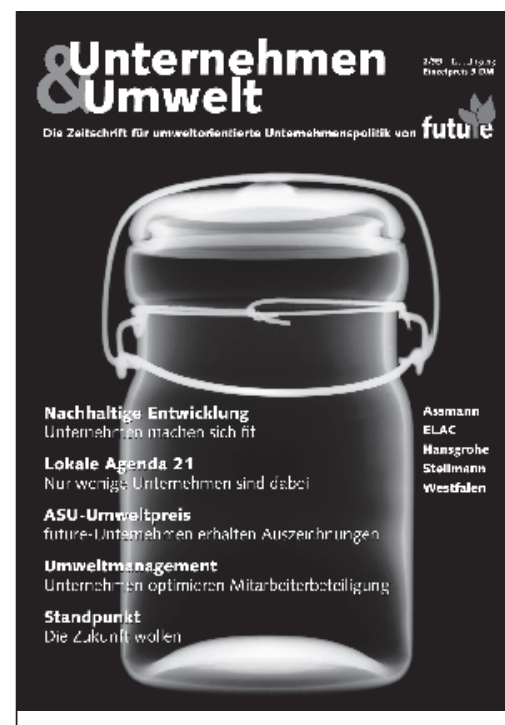

Unternehmen \& Umwelt

- ist die Zeitschrift für umweltorientierte Unternehmenspolitik von future e.V.,

- berichtet über den neuesten Stand zum betrieblichen Umweltmanagement in Theorie und Praxis,

- bietet vier Mal im Jahr ein Schwerpunktthema, Unternehmensbeispiele, Literaturund Veranstaltungshinweise und aktuelle future-Nachrichten.

Zum Beispiel Heft 2/99:

\section{Nachhaltigkeit}

- Nachhaltige Entwicklung: Unternehmen machen sich fit.

- Lokale Agenda 21: Nur wenige Unternehmen sind dabei.

- Betrieblicher Umweltschutz: Assman, ELAC, Hansgrohe, Steilmann.

- Umweltmanagement: Unternehmen optimieren die Mitarbeiterbeteiligung.

Fordern Sie Ihr kostenloses Probeexemplar an:

Fon: 0234 - 97995 - 13

Fax: 0234 - 97995 - 14

future e.V. Büro Bochum

Am Varenholt 123

44797 Bochum

email: future@umis.de
Die Belange des Schutzes der natürlichen Ressourcen werden an Hand der Richtlinien der European Foundation for Quality Management (EFQM) für die Selbstbewertung erfasst (1) und in das vorhandene Qualitätsmanagementsystem eingegliedert. In diesem Zusammenhang ist zu betonen, dass das EFQM-Selbstbewertungssystem im gesamten Konzern eingefiuhrt wird. Die Selbstbewertung schärft das Bewusstsein der Führungskräfte in Bezug auf alle erfolgsrelevanten Faktoren. Der ganzheitliche Ansatz ist für die Annäherung an die Nachhaltigkeit sehr förderlich. Diese Systeme bilden eine Basis, auf der weitere Subsysteme und Instrumente aufgestellt wurden. Besonders zu erwähnen sind hier die Systeme für das Abfallmanagement, Umweltcontrolling und das Flottenmanagement. Darüber hinaus wurden spezielle Werke für die Unterstützung der Verantwortlichen für die Produktgestaltung und für den Beschaffungsprozess entwickelt.

3. Einbeziehung der Umweltrelevanz der Produkte und Dienstleistungen.

In den kommenden Monaten und Jahren werden systematisch alle wichtigen Dienste der Deutschen Telekom auf ihre Umweltrelevanz untersucht. Es geht darum festzustellen, welche Dienste in welcher Form einen Beitrag zur Steigerung der Ressourceneffizienz der Gesellschaft beitragen können. Das Ziel ist dann, diese Erkenntnisse in die Produktstrategie einfließen zu lassen.

4. Aktive Beteiligung des Unternehmens an dem Dialog mit der Gesellschaft über den richtigen Weg zur Nachhaltigkeit.

Wie eingangs festgestellt, leben wir in einer nicht nachhaltigen Gesellschaft, dennoch sind wir der Überzeugung, dass das Modell der Nachhaltigkeit eine Alternative für die Zukunft darstellt. Nachhaltigkeit kann nicht mit Hilfe des Ordnungsrechtes in die Gesellschaft eingeführt werden. Zwar sind förderliche Rahmenbedingungen notwendig, aber die Entscheidung hat die Gesellschaft insgesamt - wenn auch wahrscheinlich nicht im Konsens aller gesellschaftlichen Gruppen - zu treffen.

Es ist davon auszugehen, dass in Zukunft aufgrund eines deutlichen Anstiegs des weltweiten Ressourcenverbrauchs nicht nur die Verknappung dieser Ressourcen, sondern auch die daraus resultierenden Emissionen zu verschärften Umweltproblemen führen werden. Beispiele sind die Erwärmung der Atmosphäre, Zunahme der Häufigkeit und Stärke von Stürmen, Verknappung des Trinkwassers oder die Zunahme der Versteppung. Auch wenn die Deutsche Telekom den größten Anteil der Umsätze in Mitteleuropa erwirtschaftet, wo die Preise für elektrische Energie für eine Weile noch fallen werden und noch keine der von Wissenschaftlern prognostizierten Probleme deutlich zu spüren sind, darf nicht verkannt werden, dass eine Neuausrichtung eines Großunternehmens ein langwieriger Prozess ist. Aus diesem Grund ist es notwendig, pro-aktiv zu handeln. Dabei nutzen wir die Vorteile aus unserer Tradition und der Tatsache, dass sicherlich in Deutschland die gesellschaftlichen Rahmenbedingungen hierfür günstiger sind als in andern Ländern. In unseren Handlungen berücksichtigen wir als global agierendes Unternehmen die weltweiten Entwicklungen.

\section{- Wirtschaftlicher Nutzen}

Den Nutzen aus unseren Aktivitäten zum Schutze der Umwelt schöpfen wir aus drei Quellen:

1. Verbesserung der internen Prozesse, Erkennung und Umsetzung von Einsparpotenzialen. Das Umweltmanagementsystem trägt dazu bei, Kosten zu sparen.

2. Einsatz des Umweltschutzes in der Kommunikation mit unseren Kunden, um eine Steigerung der Akzeptanz unserer Produkte und Dienstleistungen zu erzielen. Wir können somit Wettbewerbsvorteile realisieren und ausbauen.

3. Die sehr guten Ergebnisse der externen ÖkoRatings, vie von oekom AG und Innovest, steigern die Attraktivität unserer Aktie. Größere Investoren, aber auch Privatpersonen berücksichtigen zunehmend ethische Aspekte bei ihren Anlage-Entscheidungen. Der eingeschlagene Weg trägt bereits deutliche Früchte. Im September 1999 wurde die Deutsche Telekom in den Dow Jones Sustainability Group Index aufgenommen. Sie ist die weltweite Leading Sustainability Company in der Telekommunikationsbranche.

Wir betrachten die bisher erzielten Erfolge als eine Verpflichtung für die Zukunft. Sie zeigen uns auch, dass wir auf dem richtigen Weg sind.

Anmerkung

(1) Vgl. European Foundation for Quality Management EFQM (Hrsg.): Bestimmung der Qualität von Unternehmensleistungen - Fragebogen-Methode, Brüssel 1995.

\section{Der Autor}

Dr. habil. Ignacio Campino ist Leiter des Zentralbereichs Umweltschutz der Deutschen Telekom.

Kontakt: Deutsche Telekom AG, Am Kavalleriesand 3, 64295 Darmstadt, Tel. 06151/83-3340, Fax -4246, E-mail: Ignacio.Campino@telekom.de 
(c) 20I0 Authors; licensee IÖW and oekom verlag. This is an article distributed under the terms of the Creative Commons Attribution Non-Commercial No Derivates License (http://creativecommons.org/licenses/by-nc-nd/3.o/), which permits unrestricted use, distribution, and reproduction in any medium, provided the original work is properly cited. 